\title{
Phase Transitions and Nanowire Formation in B2 and B19 NiTi Metallic Alloys
}

\author{
Maxwel Gama Monteiro Junior'; João Paulo Almeida de Mendonça ${ }^{\mathbf{1}}$; Douglas Martins Vieira da Silva ${ }^{\mathbf{1}}$; \\ Pablo Zimmerman Coura ${ }^{\text {; }}$ Sidiney de Andrade Leonel ${ }^{1}$; \\ Fernando Sato ${ }^{1}$ \\ 1 Universidade Federal de Juiz de Fora, Dep. Física, Instituto de Ciências \\ Exatas, Juiz de Fora, Minas Gerais, Brazil. \\ maxweljr@gmail.com
}

\begin{abstract}
Molecular dynamics simulations were performed on two specific phases of the binary alloy known as Nitinol $\left(\mathrm{Ni}_{50} \mathrm{Ti}_{50}\right)$ : the high temperature $\mathrm{B2}$ phase (austenite) and the orthorhombic B19 phase (martensitic). Statistical results were obtained on the formation of nanowires by mechanical stretching for both phases, under constant temperature conditions. Dynamical stabilities of both phases modeled by a tight-binding potential (second moment approximation) was verified from their pair distribution functions. A general behavior of phase transition from B2 to B19 was observed during simulations, for both cases of initial conditions for dynamics (starting as B2 and starting as B19).
\end{abstract}

Index Terms-Phase Transition; Shape Memory; Nanowire.

\section{INTRODUCTION}

$\mathrm{N}$ owadays, phenomena at nanoscale have been widely used tools for technological development. In this manner, many materials have been studied from both an experimental and theoretical point of view, in order to consolidate our knowledge about them [1].

In 1962, Wiley [2] found that a binary allow composed of nickel and titanium (NiTi, commonly called of nitinol) exhibit a shape recovery effect. The materials which have this property are called of shape-memory alloys (SMAs) and their remarkable mechanical properties upon loading followed by heating are known as shape memory effect (SME).

Nitinol alloys have been widely studied due to their potential applications in materials science. They present good mechanical properties and stability under stressful conditions, and can be produced by a wide range of techniques [3-5]. A full atomic scale understanding of the shape-memory effect in $\mathrm{NiTi}$ is still developing, a problem particularly relevant to ongoing work in shape-memory devices for use in mesoscale and nanoscale systems.

In the general way the alloy nitinol can be characterized according to their crystallographic phase. It can be either austenite having a cubic B2 structure for high temperature or martensite B19, B19' and R phase (they are obtained by

'Maxwel Gama - maxweljr@gmail.com distortion of the B2 structure) for low temperature and which are energetically more stable [6-7]. This transition represents a phenomenon of long range ordering below the critical temperature $T_{t}$, with elastic strain acting as an order parameter on the formation of domains with the same structural properties and energy, but different directions [8].

Nanostructures of NiTi and other alloys based on it with SME have been widely studied by a variety of experimental procedures. Phase transformations for these alloys are dependent on many factors and have been experimentally investigated under many conditions, such as chemical composition, thermal and mechanical history [5,7-9]. X-Ray Diffraction (XRD) and Differential Scanning Calorimetry (DSC) are commonly used to determine stability properties and phase transformations on the alloys. The phase transformations themselves are usually obtained by means of temperature variation (thermoelastic transformations), however the use of High Pressure Torsion (HPT) [8] and pressure changes at room temperature (pressure-induced transformations) [9] have also been reported.

Nanowires (NWs) have been the target of many experimental and theoretical works due to their interesting properties and potential applications [10]. The effect of torsion on the mechanical properties of NiTi NWs of diverse shapes have been reported [11], but the specific conditions of their formation, specially as the NW thickness decreases, possibly up to the formation of Linear Atomic Chains (LACs), are still elusive.

We investigate the formation of NWs performing molecular dynamics (MD) simulations, with both austenite (B2) and B19 phase as initial configurations at room temperature. From experimentally techniques, the B2 phase can be obtained at room temperature either from quenching or by the addition of Fe to the structure [9]. In the simulations the identification of the different structural phases are made possible by their Pair Distribution Function (PDF).

\section{METHODOLOGY}

The problem of studying a solid from its constituting atomic species, as well as their collective behavior, presents several degrees of freedom. From a macroscopic point of view, temperature changes, tension, stress and crystallographic 
orientation may drastically influence the dynamical trajectory of a system. Thus, for a theoretical investigation, we require a model that can properly reproduce all these aspects, while also being viable as a computational tool, in the sense of its efficiency as software.

In this work the potential energy between particles is calculated using a potential based on the Tight-Binding Second Moment Approximation (TB-SMA) scheme where is taken into account the electronic band structure and the local density of states (LDOS). It has been successfully applied to study the structure of different atomic species as well as metallic alloys [12-14], and used in comparative studies, confirming its capability [1,15-18].

The total cohesive energy of the system is given by:

$$
E_{C}=\sum_{i}\left(E_{R}^{i}+E_{A}^{i}\right)
$$

Where:

$$
\begin{aligned}
& E_{R}^{i}=\left[\sum_{j} A_{\alpha \beta} e^{-p_{\alpha \beta}\left(\frac{r_{i j}}{r_{0}^{\alpha \beta}}-1\right)}\right] \\
& E_{A}^{i}=\left[\sum_{j} \xi_{\alpha \beta}^{2} e^{-2 q_{\alpha \beta}\left(\frac{r_{i j}}{r_{0}^{\alpha \beta}}-1\right)}\right]^{1 / 2}
\end{aligned}
$$

The first term is the repulsive interaction (Born-Mayer ionion repulsions) and the second term is the band energy. The index $\alpha \beta$ correspond to the atomic species $(\mathrm{Ni}-\mathrm{Ni}, \mathrm{Ni}-\mathrm{Ti}$, or $\mathrm{Ti}-\mathrm{Ti}), r_{0}^{\alpha \beta}$ is the first neighbor distance between the atoms in the $\alpha \beta$ lattice (experimental value), ${ }^{r_{i j}}$ is the distance between atoms $\mathrm{i}$ and $\mathrm{j}$. The other parameters were fitted to the experimental values of cohesive energy, lattice parameters, and elastic constants at $\mathrm{T}=0 \mathrm{~K}$ for $\mathrm{B} 2$ phase NiTi, taking into account equilibrium conditions. This method is described with more details in the references [19-20]. The parameters used for this work are detailed in table 1 .

TABLE 1: Potential parameters for the Ti-Ni, Ti-Ti, and Ni-Ni interactions modeled by TB-SMA.

\begin{tabular}{|c|c|c|c|}
\hline Parameter & $\mathrm{Ti}-\mathrm{Ni}$ & $\mathrm{Ti}$ & $\mathrm{Ni}$ \\
\hline$E$ (eV/atom) & $5.02^{\mathrm{a}}$ & $4.85^{\mathrm{b}}$ & $4.44^{\mathrm{b}}$ \\
\hline$r_{0}$ (Angstrom) & $2.607^{\mathrm{a}}$ & $2.95^{\mathrm{a}}$ & $2.49^{\mathrm{b}}$ \\
\hline$A(\mathrm{eV} /$ atom $)$ & $0.3^{\mathrm{a}}$ & $0.153^{\mathrm{a}}$ & $0.104^{\mathrm{a}}$ \\
\hline$p$ & $7.9^{\mathrm{a}}$ & $8.62^{\mathrm{b}}$ & $16.999^{\mathrm{b}}$ \\
\hline$\xi(\mathrm{eV} /$ atom $)$ & $2.48^{\mathrm{a}}$ & $1.879^{\mathrm{a}}$ & $1.591^{\mathrm{a}}$ \\
\hline$q$ & $3.002^{\mathrm{a}}$ & $2.390^{\mathrm{b}}$ & $1.189^{\mathrm{b}}$ \\
\hline \multicolumn{4}{|c}{${ }^{\mathrm{a}}[20],{ }^{\mathrm{b}}[19]}$. \\
\end{tabular}

To numerically solve the time evolution of the system, we use Verlet's algorithm [10,19], with a time step of $\Delta t=2 \mathrm{fs}$. The positions and velocities are calculated, respectively, using the following equations:

$$
\begin{aligned}
& \vec{r}_{i}\left(t_{n+1}\right)=\vec{r}_{i}\left(t_{n}\right)+\vec{v}_{i}\left(t_{n}\right) \Delta t+\frac{1}{2} \vec{a}_{i}\left(t_{n}\right) \Delta t^{2} \\
& \vec{v}_{i}\left(t_{n+1}\right)=\vec{v}_{i}\left(t_{n}\right)+\frac{1}{2}\left(\vec{a}_{i}\left(t_{n}\right)+\vec{a}_{i}\left(t_{n+1}\right)\right) \Delta t
\end{aligned}
$$

Where $\vec{a}$ is the acceleration acting on the atom and is determined calculating the gradient of the cohesive energy $E_{C}$ [19].

To describe structural phases in our simulations during time integration, we use the Radial Pair Distribution Function (RDF). It represents the probability of finding a particle a distance $r$ away from a given reference particle, relative to that of an ideal gas.

This PDF is usually determined by calculating the distance between all particle pairs and binning them into a histogram. In this sense, it is a radial pair distribution function. The histogram is then normalized with respect to the ideal gas, whose particle histograms are completely uncorrelated.

In the three dimensional case, this normalization is given by:

$$
g(r)=4 \pi r^{2} \rho d r
$$

Where $\rho$ is the number density of the system (number of particles per unit volume), and $4 \pi r^{2} d r$ is the volume of an infinitesimally thick spherical shell centered on the reference particle, with radius $r+d r$ [21].

The simulation of the nanowires formation was taken stretching both ends of the structure. The edges have been considered frozen during stretching (Figure 1), to mimic the initial crystallographic orientation [22].

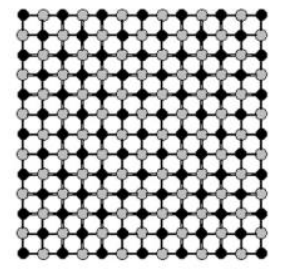

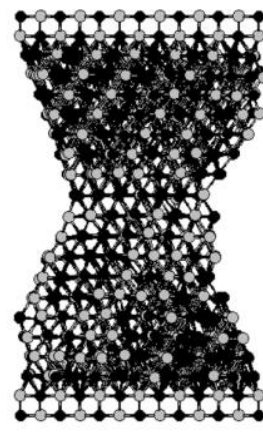

(b)

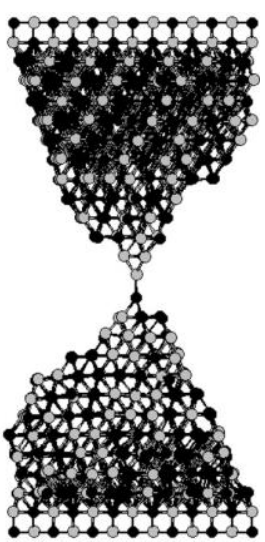

(c) (a)
Figure 1: Frames of MD evolution to 0 (a), 1437 (b) and 2418 (c) steps in a particular setup. At the top and bottom of the figures, it is possible to see the frozen atoms during the dynamics that are pulled. (c) is an example of a linear chain formation. 
The Molecular Dynamics (MD) computer program is written by the authors, both on FORTRAN and on CUDA C programming languages, as well as the software used in building the profiles from pair distribution functions. The CUDA C software was written specifically for parallel running on graphics processing units (GPU) [23].

\section{RESUlTS AND DISCUSSION}

We conducted a statistical counting for the study of nanowires formation by mechanical stretching at specific directions. We performed a total of three hundred simulations, at $300 \mathrm{~K}$, and by stretching an initial structure (at either B2 or B19 phases initially) to a loading rate $1 \mathrm{~m} / \mathrm{s}(0.5 \mathrm{~m} / \mathrm{s}$ at each apex), until NW formation and subsequent rupture of the structure.

NWs were classified into three groups: the first is the LAC that was defined by a single suspended atom held by two bonds from each side; the second is the other types of nanowires defined, as example as four paired atomic bonds holding the rest of the structure together under stress [24]; and the third any configuration that is not featured as LAC or other NWs. Overall, in the last group, rupturing occurs by the displacement of entire crystallographic planes, with dimensions close to that of the initial condition atomic layers, and represent the abrupt break of structure.

We present the statistical results on LAC formation in Table 2. Pulling directions were chosen as [001], [110] and [111]. For each direction, 100 simulations were ran, 50 with each phase being the initial condition.

TABLE 2: Number of occurrence of the LAC formation according to the total simulations for each crystallographic direction and structural phase.

\begin{tabular}{|c|c|c|}
\hline $\begin{array}{c}\text { Crystallographic } \\
\text { Direction }\end{array}$ & Initial Phase & Initial Phase \\
\cline { 2 - 3 } & $\mathrm{B} 2$ & $\mathrm{~B} 19$ \\
\hline$[001]$ & $3 / 50$ & $4 / 50$ \\
\hline$[110]$ & $5 / 50$ & $9 / 50$ \\
\hline$[111]$ & $13 / 50$ & $18 / 50$ \\
\hline
\end{tabular}

TABLE 3: Number of occurrence of the formation of other types of nanowires for each crystallographic direction and structural phase. This count does not include the formation of LAC, which is presented in TAB 2.

\begin{tabular}{|c|c|c|}
\hline $\begin{array}{c}\text { Crystallographic } \\
\text { Direction }\end{array}$ & Initial Phase & Initial Phase \\
\cline { 2 - 3 } & $\mathrm{B} 2$ & $\mathrm{~B} 19$ \\
\hline$[001]$ & $8 / 50$ & $11 / 50$ \\
\hline$[110]$ & $9 / 50$ & $12 / 50$ \\
\hline$[111]$ & $16 / 50$ & $20 / 50$ \\
\hline
\end{tabular}

On Table 3 we present the results for NWs formation, which does not include LAC formation. Cases that do not fit in Tables 2 and 3 are events that make up the third group. These are the cases where there was a sudden rupture of the structure, representing the majority of simulations.

Both the LAC and NW formation is most frequently observed along [111] direction for the two phases of NiTi and it is more frequent to the initial phase B19. This result is in good agreement to the fact that martensitic phases undergo less internal stresses than the high temperature austenite, due to the spontaneous self organization of the lattice at this stage [8,9].

To obtain information about the phase transitions occurring during the dynamics, we plot the structure's Radial Pair Distribution Function, as described previously.

We set up the super cell to B2 and the unit cell to B19 structures, using information in the reference [6], in complete agreement with references [19] and [20]. The values of lattice parameters and the super cell angles to $\mathrm{B} 2$ are: $\mathrm{a}=2.937 \AA$, $\mathrm{b}=4.154 \AA, \mathrm{c}=4.154 \AA, \alpha=90^{\circ}, \beta=90^{\circ}$ and $\gamma=90^{\circ}$. For the case of $\mathrm{B} 19$, the parameters are: $\mathrm{a}=2.637 \AA, \mathrm{b}=4.557 \AA$, $\mathrm{c}=4.170 \AA$, $\alpha=90^{\circ}, \beta=90^{\circ}$ and $\gamma=90^{\circ}$. These cells are illustrated in Figure $1 \mathrm{a}$ and $1 \mathrm{~b}$. The characterization of these two structures was made by PDF profile and is shown in Figure 2.

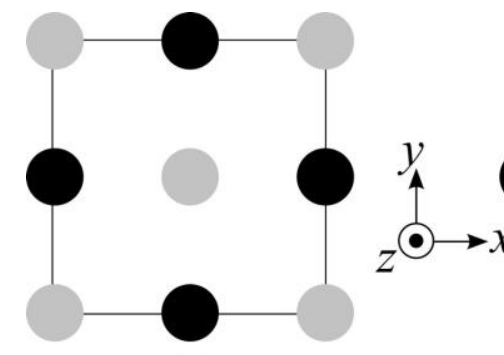

(a)

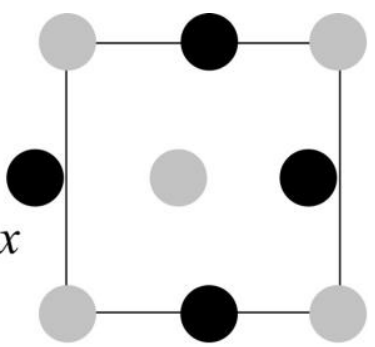

(b)

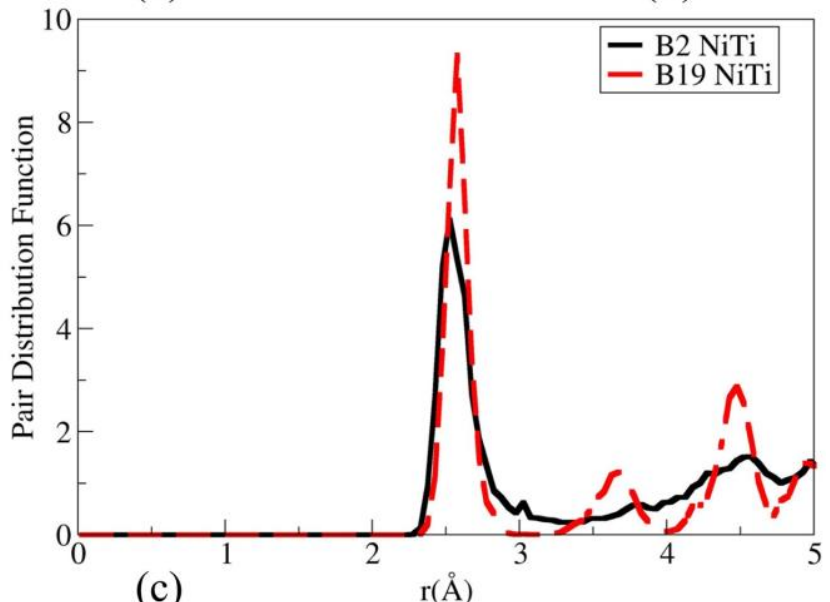

Figure 2: (a) The super cell for NiTi B2 [001] direction and (b) the unit cell for NiTi B19 [001] direction. (c) PDF profile for B2 and B19 NiTi phases.

The PDF profile curves clearly show differences between the two structures studied. The B19 phase has two apparent peaks in the second and third neighbor, while B2 phase for these peaks are less evident. This simple difference allows us to identify the two different phases B2 and B19 during dynamics [6,20].

Interestingly, every simulation at room temperature has shown a one direction transformation from B2 into the B19 phase, simultaneously across the whole structure. The average number of molecular dynamics steps for this transformation to occur was counted to be: 


$$
P=(50 \pm 34) \text { steps }
$$

For our chosen time step of 2 fs, $\mathrm{P}$ corresponds to an average of $100 \mathrm{fs}$ before the phase transformation took place. For the initial B19 phase condition, no similar phenomenon was observed, and the structure preserved its overall shape except for expected defects due to stress. A pair of simulations was repeated for both initial conditions, but at a temperature of $400 \mathrm{~K}$, at which the $\mathrm{B} 2$ phase modeled by TB-SMA retains its form in unconstrained molecular dynamics [6,20]. Results are shown in Figures 3 and 4. Even then, the nanostructures swiftly favored the transition to B19 under the mechanical stretching.

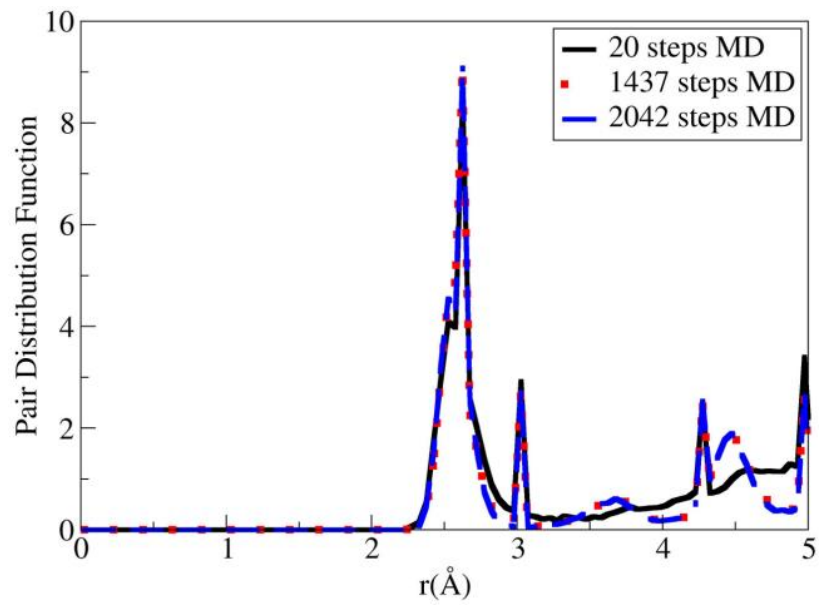

Figure 3: PDF for B2 initial conditions dynamics, pulling through [001] at $400 \mathrm{~K}$, showing the transition to B19 during molecular dynamics. The dark line is at 20 time steps (2fs per step) while the clearer ones at 1437 and 2042 steps, respectively.

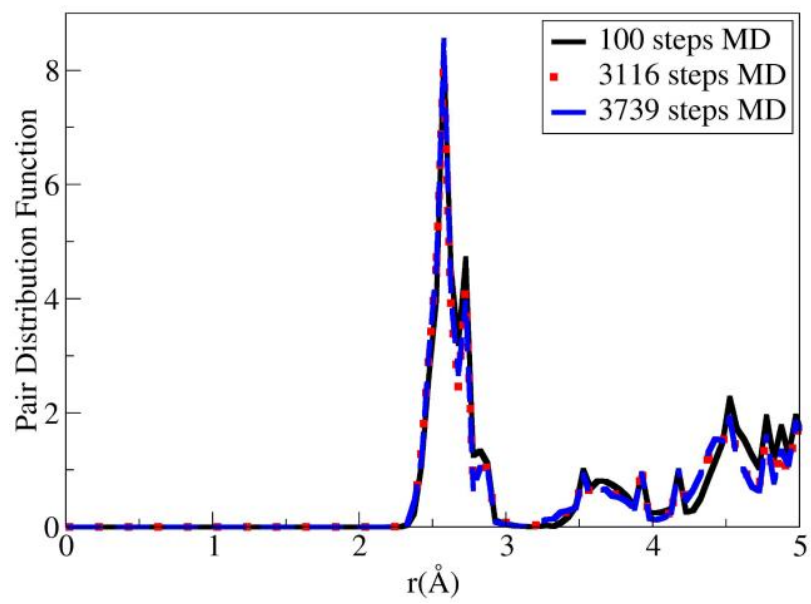

Figure 4: PDF for B19 initial conditions dynamics, pulling through [001] at $400 \mathrm{~K}$, showing that no transitions appear during molecular dynamics. The dark line is at 100 time steps ( 2 fs per step) while the clearer ones at 3116 and 3739 steps, respectively.

The phase transformations reported suggest that stress along the outmost layers of the B2 nanostructure is inducing a change to an orthorhombic phase even under constant temperature conditions. For such small samples (only dozens of atoms thick) surface energy is a huge factor to take into play, and any dynamical feature introduced to the external layers therefore may produce unpredictable consequences.

Literature on pressure-induced transformations indicates that the austenite NiTi phase, as well as its related alloys, indeed undergoes transition to B19 under high pressures $[9,25]$. However, the molecular dynamics tool used in this work consider a canonical ensemble (NVT) and the stress/strain dependence of the phase transformation can only be inferred from the fact that NWs were more probable to occur from the B19 phase, matching the experimental data showing it minimizes strain energy in relation to the high symmetry B2 phase [4-9].

\section{Conclusion}

We studied the formation of NWs in equi-atomic NiTi at room temperature, also taking into account its phase transitions under rupture, through molecular dynamics simulations. Across all pulling directions considered, the B19 Phase was shown to be the most favorable for NW formation.

An unexpected and constant phase transition behavior, from B2 to B19, was observed, and it is believed that there are surface effects related to stress at play. The only correlation which can be established so far is that of the B19 being more probable to form NWs than B2, while also being the preferred symmetry of equi-atomic NiTi structures under mechanical stretching across a single direction. Further investigation is required, especially considering stress/strain dependent phenomena on the nanoscale.

The authors acknowledge support from the Brazilian Agencies CAPES, FAPEMIG, CNPq, and UFJF/PROPGPI.

\section{REFERENCES}

[1] A. P. Sutton. Electronic Structure of Materials. Oxford University Press Inc., Oxford, 1996.

[2] W.J. Buehler, R.C. Wiley, trans. Am. Soc. Metal. 55 (1962) 269

[3] Otsuka. K and Wayman C. Shape Memory Materials. Cambridge University. Press, New York, 1998

[4] T.W. Duerig and A. R. Pelton. Ti - Ni Shape Memory Alloys. ASM International, 1994.

[5] K. K. Mahesh, F. M. B. Fernandes, and G. Gurau. Advanced Materials Research 123-125 1007-1010, 2010.

[6] Xiangyang Huang, Graeme J. Ackland, and Karin M. Rabe. Nature Materials, 2:307 - 311, 2003.

[7] Pasturel A., Colinet C., Nguyen Manh D., Paxton A. T., and van Schilfgaarde M. Phys. Rev. B, 52:15176, 1995.

[8] S. Sarkar, X. Ren, and K. Otsuka. Physical Review Letters 95, 205702, 2005.

[9] A.S. Ferreira, P. R. Rovani, and A.S. Pereira. Journal of Applied Physics 117, $075901,2015$.

[10] F. Sato, M. J. Lagos, P.A.S. Autreto, D. Ugarte, and D.S. Galvão. Physicae 8, UNICAMP, 2009.

[11] C.D. Wu, P.H. Sung, T.H. Fang. J. Mol. Model. 19(4), 1883-90,2013.

[12] F. Sato, A. S. Moreira, J. Bettini, S. O. Dantas, P. Z. Coura ,D. Ugarte, D. S. Galvão. Phys. Rev. B, 74:193401, 2006.

[13] P. Z. Coura, S. B. Legoas, A. S. Moreira, F. Sato, V. Rodrigues, S. O. Dantas,D. Ugarte, and D. S. Galvão. Nano Letters, 4:1187, 2004.

[14] J. Bettini, F. Sato, P. Z. Coura, S. O. Dantas, D. S. Galvao, and D. Ugarte. Nature Nanotechnology, 1:182, 2006. 
[15] Q. Pu, Y. Leng, L. Tsetseris, H.S Park, S.T. Pantelides, and P.T. Cummings. J. Chem. Phys., 126:144707, 2007.

[16] F. Ducastelle and F. Cyrot-Lackmann. J. Phys. Chem. Solids, 32:285, 1971.

[17] F. Cyrot-Lackmann. Surf. Sci., 15:1235, 1968.

[18] F. Cyrot-Lackmann. J. Phys. Chem. Solids, 29:1235, 1968.

[19] F. Cleri and V. Rosato. Phys. Rev. B, 48:22 33, 1993.

[20] W.S. Lai and B.X. Liu. J. Phys.: Condens. Matter, 12:L53-L60, 2000..

[21] Chandler, D.. Introduction to Modern Statistical Mechanics. Section 7.3, Oxford University Press. (1987)

[22] P. Z. Coura, S. B. Legoas, A. S. Moreira, F. Sato, V. Rodrigues, S. O. Dantas, D. Ugarte, and D. S. Galvão. Nano Letters, 47 (1187-1191), (2004).

[23] Jason Sanders, Edward Kandrot. CUDA by example: an introduction to general purpose GPU programming. Pearson Education, Inc., Boston, 2010.

[24] N. Agrait, A. L. Yeyati, and J. M. van Ruitenbeek, Phys.Rep. 377, 81 (2003).

[25] V. G. Pushin, R. Z. Valiev, E. Z. Valiev, N. I. Kourov, N. N. Kuranova, V.V. Makarov, A. V. Pushin, and A. N. Uksusnikov, Phys. Met. Metall.113(3), 256 (2012). 